\title{
Varying matrix effects for elemental analysis identified from groundwater in the Barnett Shale
}

\author{
D. D. Carlton Jr. ${ }^{1}$ - B. E. Fontenot ${ }^{2,5}$ - Z. L. Hildenbrand ${ }^{3}$ T. M. Davis ${ }^{1}$. \\ J. L. Walton ${ }^{4}$ K. A. Schug ${ }^{1}$
}

Received: 2 March 2014/Revised: 25 February 2015/Accepted: 15 March 2015/Published online: 9 April 2015

(C) Islamic Azad University (IAU) 2015

\begin{abstract}
The quality of analytical measurements can be influenced by the matrix of the sample of interest. The knowledge of the sample matrix allows for appropriate sample preparation, instrumental parameters, and quantification methods in an effort to achieve accurate results. Matrix matching can be difficult when sampling across various water sources with the possible introduction of unknown endogenous contaminants due to various degrees of land use, urbanization, and energy exploration, likely playing a factor. The degree of matrix effects in inductively coupled plasma-optical emission spectroscopy for nineteen metals from twenty groundwater samples across North Texas was assessed using a standard addition method. Matrix effects were characterized in collected groundwater samples (a) with no pretreatment, (b) after reversed-phase solid-phase extraction of possible organic contaminants, and (c) for a matrix of organic material retained on the reversed-phase sorbent. It was found that without any extraction treatment, only $54 \%$ of all measurements
\end{abstract}

Electronic supplementary material The online version of this article (doi:10.1007/s13762-015-0803-4) contains supplementary material, which is available to authorized users.

\section{K. A. Schug}

kschug@uta.edu

1 Department of Chemistry and Biochemistry, The University of Texas at Arlington, Arlington, TX 76019, USA

2 Independent Consultant, Arlington, TX, USA

3 Inform Environmental LLC, Dallas, TX 75206, USA

4 SWCA Environmental Consultants, Arlington, TX 76006, USA

5 Present Address: Water Quality Protection Division, United States Environmental Protection Agency, Dallas, TX 75202, USA experienced no matrix effect. After extracting unknown organic sample constituents, an increase to $74 \%$ of measurements showing no matrix effect was recorded. Reconstituting the extracted organic sample matrix found this fraction to be a significant source of the deviated results with only $13 \%$ experiencing no matrix effect. Results for the metals investigated are also discussed, along with correlations to water quality parameters such as turbidity, total dissolved solids, and salinity.

Keywords Inductively coupled plasma-optical emission spectroscopy $\cdot$ Solid-phase extraction $\cdot$ Sample preparation - Unconventional drilling

\section{Introduction}

Accuracy in the quantification of metals in environmental samples, whether water, soil, or air, is of the upmost importance for the safety of living organisms. The significance of these measurements is made evident by the 200 series of test methods by the United State Environmental Protection Agency (EPA) which is nearly exclusive for elemental analysis and includes extensive sampling, analysis, and verification guidelines for each. Standardization of these methods is important to ensure interlaboratory reproducibility and accuracy for these samples, which will contain varying degrees of complexity in the relative mixtures of compounds and ions. The complex milieu of constituents other than the analyte of interest comprises what is referred to as the sample matrix (Thomsen et al. 2006). The matrix can be highly variable depending on dissolved organic and inorganic matter, $\mathrm{pH}$, turbidity, or even sample source. Depending on the constituents of the sample matrix, a measured analyte signal 
can be decreased, enhanced, or unaffected relative to how it responds in a pure form and depending on the chosen analyses.

Simultaneous multi-metal analysis for metals in molecular, atomic, or ion form has become dominated by inductively coupled plasma (ICP) techniques (Olesik 1991), coupled with either mass spectrometry (MS) or optical emission spectroscopy (OES). ICP-MS has superior sensitivity over OES measurements, but this sensitivity limits the possible applications. With the proper measurement wavelength selection and viewing mode, ICP-OES has the capability of measuring major, minor, and trace elements in solution and boasts up to ten orders of magnitude in analytical working range (PerkinElmer Inc. 2013). ICP-OES is also unaffected by isobaric ions, a common consideration in ICP-MS. To help ensure the acquisition of accurate data with ICP-OES (Olesik 1996), instrumental parameters such as proper wavelength selection, sample aerosol formation from the nebulizer (Pereira et al. 2012), plasma temperature (Groh et al. 2009), and emission viewing mode (Silva et al. 2003) must be carefully optimized. With careful consideration, ICP-OES is an accurate and quite versatile technique for multi-elemental analysis.

Since the sample matrix can affect analyte response (Olesik 1996), the degree of matrix effect should be assessed. Matrix effects can be determined by comparing an analyte's response in the sample of interest to that of an analyte standard in a "matrix-free" environment (Nguyen et al. 2011). Here, we define a significant matrix effect as that which elicits a response less than $80 \%$ or greater than $120 \%$ of a matrix-free calibration standard (US EPA 1999).

Matrix effects in spectroscopy can be classified into three groups: physical, chemical, and spectral (Thomsen and Schatzlein 2006). Physical effects include changes due to a sample's viscosity, density, surface tension, and concomitant elements and their concentration. A chemical source for matrix effects result from the analyte forming a new compound with different characteristics. High temperatures of the plasma in ICP-OES typically decompose many compounds into its elemental constituents, which limits this effect. However, precipitation of the analyte before analysis, due to the formation of an insoluble salt in solution, can be problematic in analyte recovery. For example, adding sulfuric acid to a sample solution containing barium will produce barium sulfate, which has a very low solubility in water (Jiang 1996; Dow Water and Process Solutions 2011). Spectral effects are caused by the overlap of emission lines of other species at the monitored wavelength. This overlap will enhance the monitored signal, resulting in a positive measurement bias or a false positive in detection (Thomsen et al. 2006). In samples containing high concentrations of copper and minimal amounts of zinc, the copper emission line at $213.853 \mathrm{~nm}$ could lead to an overestimation in the response to the Zinc emission line at 213.856 (US EPA 2007). Of the thirty-two elements monitored at trace levels in the US EPA Method 2007, only ten elements are listed to have no spectral interferences (US EPA 2007; Kramida et al. 2013).

Ensuring the accuracy of elemental measurements in groundwater has been important for researchers and governmental agencies attempting to characterize the quality of aquifers and other water supplies throughout the country. Databases of measurements are often useful as benchmarks in situations where ground contamination events may have occurred. Recently, water in the Barnett Shale formation in North Texas has been of interest, given the expansion of unconventional drilling activities in the region (Fontenot et al. 2013). Throughout the past 10 years, the formation, spanning $48,000 \mathrm{~km}^{2}$, has become one of the most heavily drilled shale formations in the USA for the extraction of natural gas. According to the Texas Rail Road Commission, over 17,000 gas wells are recorded in the area, with the most rapid increase in the number of new wells attributed to advances in horizontal drilling and hydraulic fracturing since 2005 (www.rrc.state.tx.us/data/ fielddata/barnettshale/pdf).

Using elemental analysis of groundwater to identify instances of natural gas drilling or hydraulic fracturing contamination has tremendous merit given that many of the metals used in unconventional drilling are well established. Various metals are used in high abundances throughout the drilling, hydraulic fracturing, and extraction process, including barium, boron, molybdenum, and zirconium (Caenn et al. 2011; Fink 2011). Even so, inorganic compounds added to drilling and fracturing fluids at the surface are not the only concern. Flowback water that is removed from the ground after hydraulic fracturing has been mixed with the sediments within the shale formation and can be enriched with naturally occurring radioactive heavy metals and brines (Osborn et al. 2011). A comparison of the aquifer's metal substituents at times prior to drilling and subsequently after would provide a compelling argument regarding the effects of the natural gas extraction process. However, in the Barnett Shale, with the great deal of industrial drilling activity over many years, it is difficult to currently design such a study. Reedy et al.(2011) were able to characterize the Trinity and Woodbine aquifers (the dominant aquifers in the Barnett Shale region) as generally good quality for organic and inorganic species based upon previous USGS reports (Welch et al. 2000) and the compiled Texas Water Development Board's (TWDB) database of groundwater measurements (Texas Water Development Board 2014) across previous decades. A recent publication by Fontenot et al. (2013) quantified As, $\mathrm{Ba}, \mathrm{Sr}$, and Se in privately owned water wells from the aquifers coinciding with the Barnett Shale after substantial 
drilling and hydraulic fracturing had occurred. The team discovered elevated levels of arsenic, selenium, and strontium in the aquifers that were statistically significant when compared to the TWDB dataset of the aquifers from 1989 to 1999 , a time before wide-spread horizontal drilling and hydraulic fracturing. It was also determined that the concentrations of $\mathrm{As}, \mathrm{Se}$, and $\mathrm{Sr}$ were higher for water wells in closer proximity to unconventional drilling sites than the sampled water wells in lesser productive regions of the shale and outer non-active, reference water well locations. Various possible mechanisms for this finding were posited.

The aim of this work performed in 2012 at the University of Texas at Arlington was to identify the similarities and variances in sample matrices which would affect elemental analyses from selected samples from the Fontenot study (2013) of the two aquifers overlying North Texas's Barnett Shale region and neighboring aquifer. Many previous studies have investigated the effects of adding easily ionizable elements (EIE) (Stewart and Olesik 1999; Agatemor and Beauchemin 2011; Fraser and Beauchemin 2009) or varying concentrations of organic acids (Nam et al. 2008; Stewart and Olesik 1998) on the response for specific elements, but we are not aware of previous studies that have investigated an unknown matrix and began to explain analyte behavior based on other resulting element concentrations and physical properties of the collected sample. By identifying sample similarity and differences, suggestions are also made for future sample preparation to increase the accuracy of ICP-OES measurements in possibly contaminated groundwater samples collected from a large geographic area.

\section{Materials and methods}

Twenty samples for this study were collected from fourteen counties across the North Texas region. All the samples were collected from private residential water wells in a manner complying with EPA groundwater well sampling criteria found in SOP \#2007 (US EPA 1999). One deviation worth noting is that all water purging and sample collection were performed with the preexisting property owner's pumping system and not separate pumps associated with the research team. Eighteen of the sampled wells were from the two aquifers within the Barnett Shale formation, and the other two well sites were used as a reference outside of the sampling area. Of the eighteen wells within Barnett Shale, sixteen were within $3.5 \mathrm{~km}$ of a natural gas well and two were approximately $20 \mathrm{~km}$ removed from natural gas extraction sites. A map of the sampling sites is shown in Fig. 1. While a considerably larger sample set would have been preferred, the detailed and lengthy treatment and analysis of each sample required the study to be limited to twenty samples.

Measurements were taken using a Shimadzu ICPE-9000 optical emission spectrometer (Shimadzu Scientific Instruments, Inc., Columbia, MD). Samples were introduced with a mini-torch nebulizer, ionized with argon plasma, and introduced into the spectrometer in the axial view. Wavelengths selected for the analysis corresponded to the default wavelength chosen through the Method Development Wizard for preliminary sample measurements prior to method optimization. Software features use elemental response factor information to estimate the concentrations of each element after a calibration with $\mathrm{Al}$ and $\mathrm{Ba}$. An average of three measurements per acquisition was selected to calculate estimated concentrations which were used for data analysis of sample response. The nineteen elements chosen for investigation were antimony, arsenic, barium, beryllium, boron, cadmium, chromium, cobalt, copper, iron, manganese, molybdenum, nickel, potassium, selenium, thallium, titanium, vanadium, and zinc. Spectral line intensities for all elements were recorded simultaneously with the large-scale charge-coupled device detector, which is characterized by a resolution of $<5 \mathrm{pm}$ at $200 \mathrm{~nm}$ in the ICPE-9000 instrument.

The method of standard addition was used to assess the degree of matrix effect that may be exhibited on the response of each metal from the well water samples. Previous work has shown standard addition to be successful not only for quantifying the influence of matrix effect but also as the most accurate of quantitative methods, when compared to techniques such as internal standardization, matrix matching, and robust plasma operating conditions (Sadler et al. 1997; Stüber and Reemtsma 2004; Dettman and Olesik 2012). A response curve for standard in DI water was generated with two additional spiked concentrations of $1 \mathrm{mg} / \mathrm{L}$ increments from a multi-metal standard solution obtained from High-Purity Standards (Charleston, SC). The elements present and their concentrations in the standard can be found in Online Resource 1. The slope of the 3-pt standard addition response curve for each element in DI water was used as our control, the matrix-free response for each metal. A 1-pt standard addition response curve was then created for each water sample before any sample preparation (besides the prescribed acidification to $2 \%$ nitric acid by volume for storage). Only one spike analysis was performed because of limited sample volume. The response curve for each element in this group with no sample preparation is denoted as "Bulk" for the remainder of this manuscript.

As a means to prepare the samples and reduce matrix components, a $15-\mathrm{mL}$ aliquot of each collected well water sample was loaded onto a Bakerbond C18 solid-phase extraction (SPE) cartridge $(500 \mathrm{mg}, 3 \mathrm{~mL}$; JT Baker; Phillipsburg, NJ). This treatment was expected to remove any hydrophobic organic sample matrix (retained on the SPE 


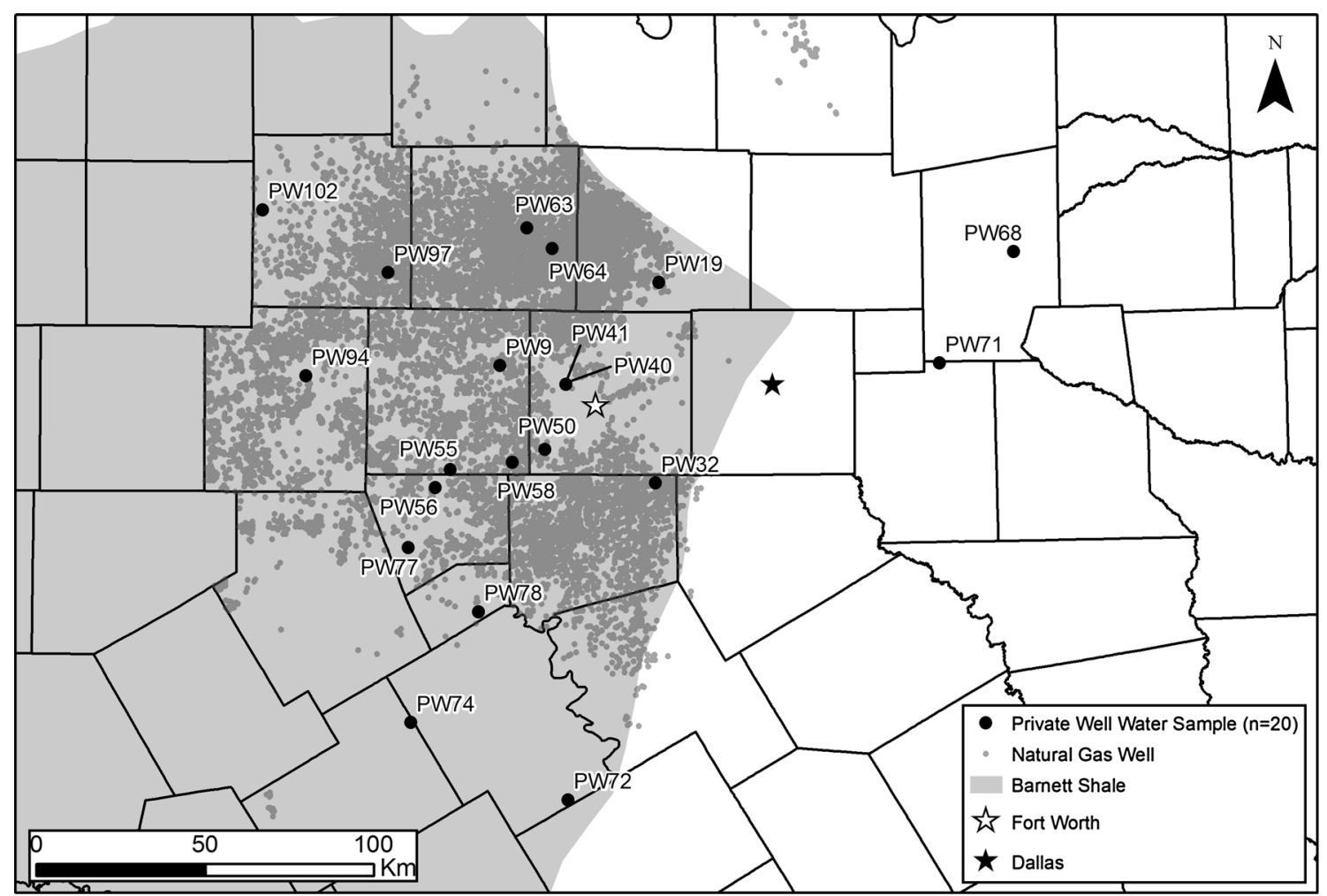

Fig. 1 Map of the sampling region from North Texas. Counties are outlined, with the Barnett Shale formation shaded in gray. Natural gas wells are represented as dark gray dots, and sampled sites are labeled with black dots. The cities of Dallas and Fort Worth are noted as points of reference

metals barium, beryllium, selenium, and vanadium showed no matrix effect present in the Bulk solution measurements of all 20 measured wells. The metals cobalt and thallium showed negative matrix effects (reduction in analyte signal in the sample vs. in DI water) for all wells, while potassium showed positive matrix effects (enhancement of analyte signal in the sample vs. in DI water) for every well measured. The survey of resulting matrix effects for Bulk sample solutions is shown in Fig. 3a. A table of specific values for each metal in each sample can be found in Online Resource 3. The concentration of each metal was calculated based upon this preparation set and corrected to account for response deviation. These values can be found in Online Resource 4.

After passing the water samples through the RP-SPE columns to retain hydrophobic organic compounds that could contribute to a sample matrix, the unretained portion of the sample aliquot, assumed to contain the metal cations, any solvents present, and other ions, was investigated for their degree of matrix present. These samples, Fraction 1, showed fewer metals affected by the groundwater matrix than did the Bulk sample solution metals, with 252 out of 342 measurements (74\%) exhibiting no matrix effects through standard addition, as shown in Fig. 3b. The metals in Fraction 1 that showed no significant matrix effects in all 
Fig. 2 Pictorial representation of the three preparation methods used to identify the sources of matrix effects. a Bulk solution is the water sample, acidified with no pretreatment. b Fraction 1 is the unretained portion of the water sample loaded onto a RPSPE cartridge. c Fraction 2 is the sample portion that was retained on the RP-SPE cartridge eluted and later rotovapped, reconstituted in DI water, and acidified
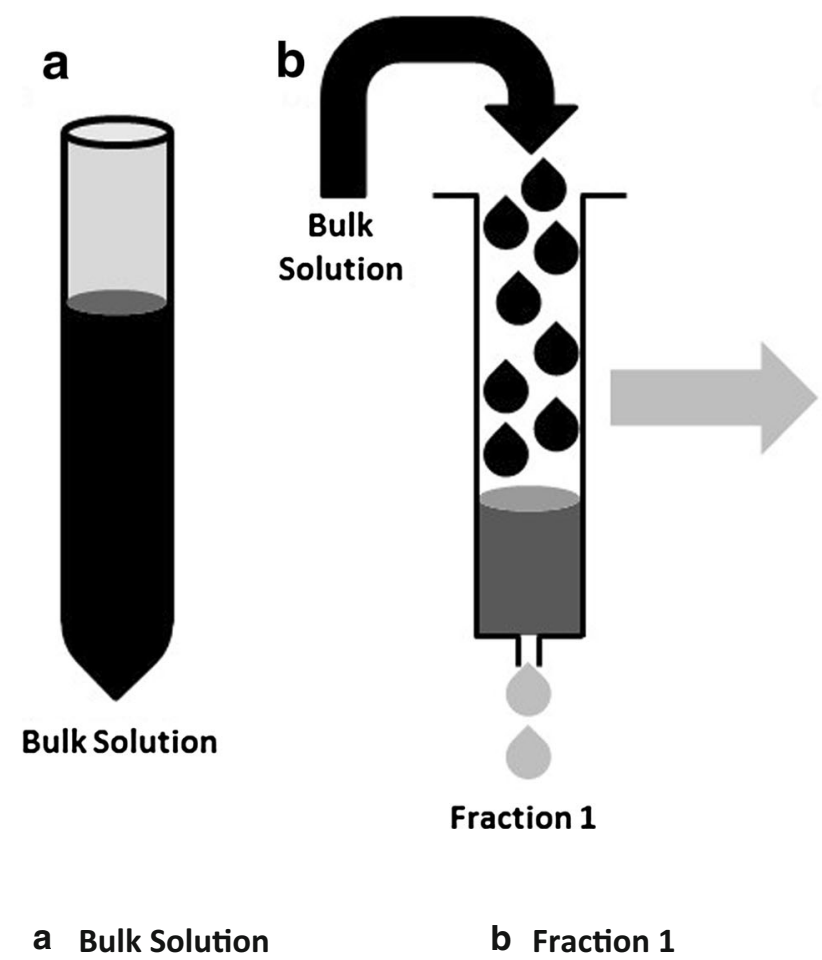

Fig. 3 Summary of the matrix effect measured for the three preparation techniques a Bulk, b Fraction 1, and c Fraction 2

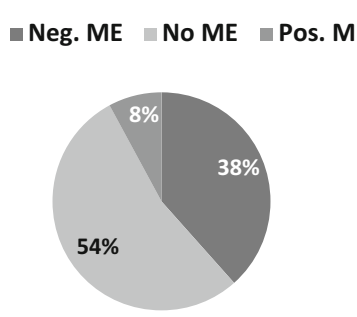

Fraction 1

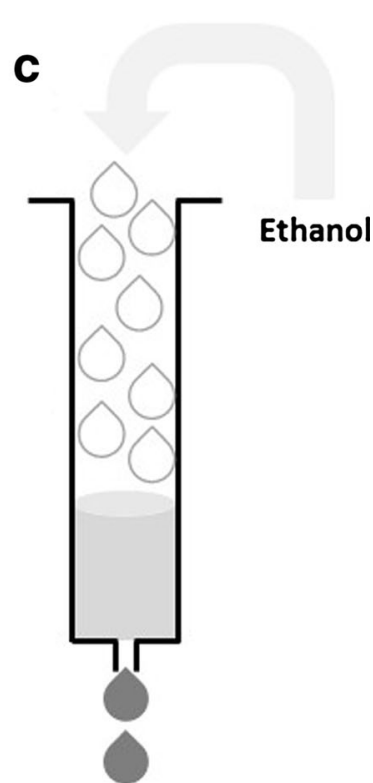

Fraction 2

\section{b Fraction 1}

\section{Fraction 2}

$\backsim$ Neg. ME $\backsim$ No ME $\backsim$ Pos. ME

$\backsim$ Neg. ME $\backsim$ No ME $\square$ Pos. ME
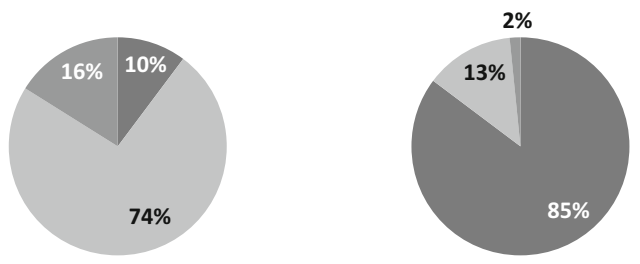

well water samples were beryllium, chromium, copper, iron, manganese, molybdenum, antimony, vanadium, and zinc. Barium, which showed no matrix effects in Bulk solution, resulted in two wells having a positive matrix effect of 24 and $26 \%$ in Fraction 1. A table of specific values for each metal in each sample can be found in Online Resource 5.

The last set of examined sample matrix was comprised of the compounds that were retained on the RP-SPE sorbent. This matrix was eluted from the sorbent, dried, and reconstituted in water. This system, identified as Fraction 2, exhibited the least amount of measurements with no matrix effect, 50 out of 380 (13\%), as shown in Fig. 3c. No metal showed an absence of matrix effect in Fraction 2 across all 20 wells. The displayed effects were quite variable for each metal and within each metal for each well. PW50 consistently returned the highest response for 18 of the 19 metals. Cobalt was the only metal where PW50 was not the most responsive, falling to second by $8 \%$ to PW32. The sample where the lowest responses were regularly observed was from PW40, yielding the lowest response in 18 of the 19 metals, and second lowest in titanium response by $1.2 \%$ behind PW77. A table of specific values for each metal in each sample can be found in Online Resource 6.

It was hypothesized that Fraction 1 would increase the number of measurements showing no matrix effects, $74 \%$, as compared to Bulk, $54 \%$. SPE is a commonly used preparation and preconcentration technique to increase the accuracy and reproducibility in elemental analysis of groundwater samples (Sah and Brown 1997; Ferreira et al. 2007). It is interesting to note the significant loss of accuracy in analyte response in Fraction 2. These comparisons indicate that the presence of matrix effects in Bulk is a combination of both Fraction 1 and Fraction 2, but to a larger extent from the organic compounds in Fraction 2. Such studies, in the context of these samples having unknown matrix compositions, reinforces the use of best practices reported previously.

\section{Cumulative sample and metal summaries}

Some applications may be interested in a sum of metal concentrations rather than that of individual metals specifically. The recovery of analyte is affected by sample 
preparation, sample introduction, and detection. Our response calculations are functions of sample introduction and detection influences. We calculate a cumulative metal response which is the average analytical response of all metals for a single sample. This metric assesses the analytical influence on measured recovery and not that of sample preparation. Based on the measurements taken of the samples as Bulk and Fraction 1, good total response recoveries are measured. The average of the cumulative metal response for the 19 metals of each of the Bulk sample solutions was $87 \pm 3 \%$ between the sampled wells. Fraction 1 showed an increased average cumulative metal response to $104 \pm 6 \%$. The consistency of the cumulative response between all the water wells shown in Bulk and Fraction 1 was not the case for Fraction 2, resulting in only a $54 \%$ average cumulative metal response with a $\pm 23 \%$ deviation between samples.

When working with multiple samples, it is important to be able to speculate the outcome of the accuracy of the measurements for a given sample preparation. We compare the averages of the absolute value of response deviation, positive or negative, from $100 \%$ analyte response to estimate how near our quantitative measurements would be to an external calibration curve for a given metal. Absolute values of each deviation allow for the two responses of 120 and $80 \%$ to give an average of $20 \%$ deviation, rather than $0 \%$. These data for each metal and three sample systems are compiled in Fig. 4a. The median deviation in response to the Bulk sample solutions was $20 \%$, which was reduced to a median deviation of only $10 \%$ in Fraction 1 . The matrix system of Fraction 2 generated a median of a $46 \%$ deviation in response across the 19 metals. An improvement in average response accuracy between Bulk and Fraction 1 was recorded for antimony, chromium, copper, iron, manganese, molybdenum, nickel, potassium, titanium, and zinc across the sampled wells. Little or no change occurred in the response for barium, beryllium, boron, cobalt, thallium, and vanadium. The greatest amount of matrix effect observed for any given metal was found in Fraction 2, except for potassium which showed the Bulk solution having the greatest average deviation of $75 \pm 23 \%$, notably in the positive direction.

Nearly all of the first-row d-block metals showed an improvement in accuracy when analyzed as Fraction 1, after being passed through the SPE cartridge. The average response deviation with one standard deviation is plotted in Fig. 4b. The average deviation of titanium through zinc in the Bulk solution was $24.7 \%$, which was reduced more than half in Fraction 1 to only $11.2 \%$ away from the response of the standard solution. Precision between each sample for the given metals also improved in Fraction 1, bettered from 6.3 to $5.2 \%$ average of the standard deviations of titanium through zinc.

\section{Water quality effects}

The average matrix effect for each water sample was compared to water quality parameters measured at the time of sample collection. Values of the physical properties anticipated to possibly affect analytical results are listed in Online Resource 7. It is notable that samples of increased salinity up to $1.49 \mathrm{~g} / \mathrm{L}$ have increased average matrix effects present (as shown in Fig. 5a). This phenomenon has been reported previously (Barth 1997; Knápek et al. 2010). Ionic species are the likely culprit. The ionic strength of the solution, whether from salts or acids/bases, can induce ion suppression or enhancement through different mechanisms (Agatemor and Beauchemin 2011). A study of ionic matrices revealed that ion suppression in $\mathrm{K}$ and $\mathrm{Cs}$ matrices across all elements studied could be counteracted with a $\mathrm{Na}$ matrix due to a Coulomb fission mechanism (Fraser and Beauchemin 2009). While samples with less than $0.4 \mathrm{~g} / \mathrm{L}$ $(n=12)$ include the highest and lowest average matrix effects per well, 32.9 and $22.2 \%$, respectively, the five samples with less than $25 \%$ average response deviation are among the eight lowest salinity measurements. The range of average absolute matrix effects at the low salinity concentrations indicates additional driving forces than just solution salinity for the response deviation.

The responses for potassium and thallium in the Bulk solution revealed similar correlations with multiple groundwater quality measurements. The least degrees of matrix effects for each element were present at the lowest concentrations of TDS and salinity for the water samples. Conversely, matrix effects for the two elements decreased as the turbidity of the solution increased. High concentrations, $\geq 10 \mathrm{mM}$, of any element efficiently ionized generally result in analyte signal suppression (Olesik 1996). These statements are supported with plots provided in Online Resource 2.

The direct relationship observed between TDS and potassium and thallium matrix effects in the Bulk solution was the opposite for selected metals in Fraction 1. Antimony, molybdenum, nickel, selenium, thallium, and vanadium all had the lowest degree of matrix effects with the highest concentrations of TDS in the original sample. On the contrary, potassium measurements continued to behave similar to the Bulk solution with the least degree of matrix effect in samples of lowest TDS, but with a weaker Spearman correlation ( $\rho=0.306$ vs. $\rho=0.756$ ).

Zinc and cadmium, two group 12 elements, had almost identical responses for each sample, with 17 of the 20 within $5 \%$ of the other in the Bulk solution. After SPE treatment, only four samples had responses within $10 \%$ of the other group 12 metals. In general, the zinc measurements show a reduced matrix effect in Fraction 1 as anticipated. Fraction 1 measurements for cadmium are quite 


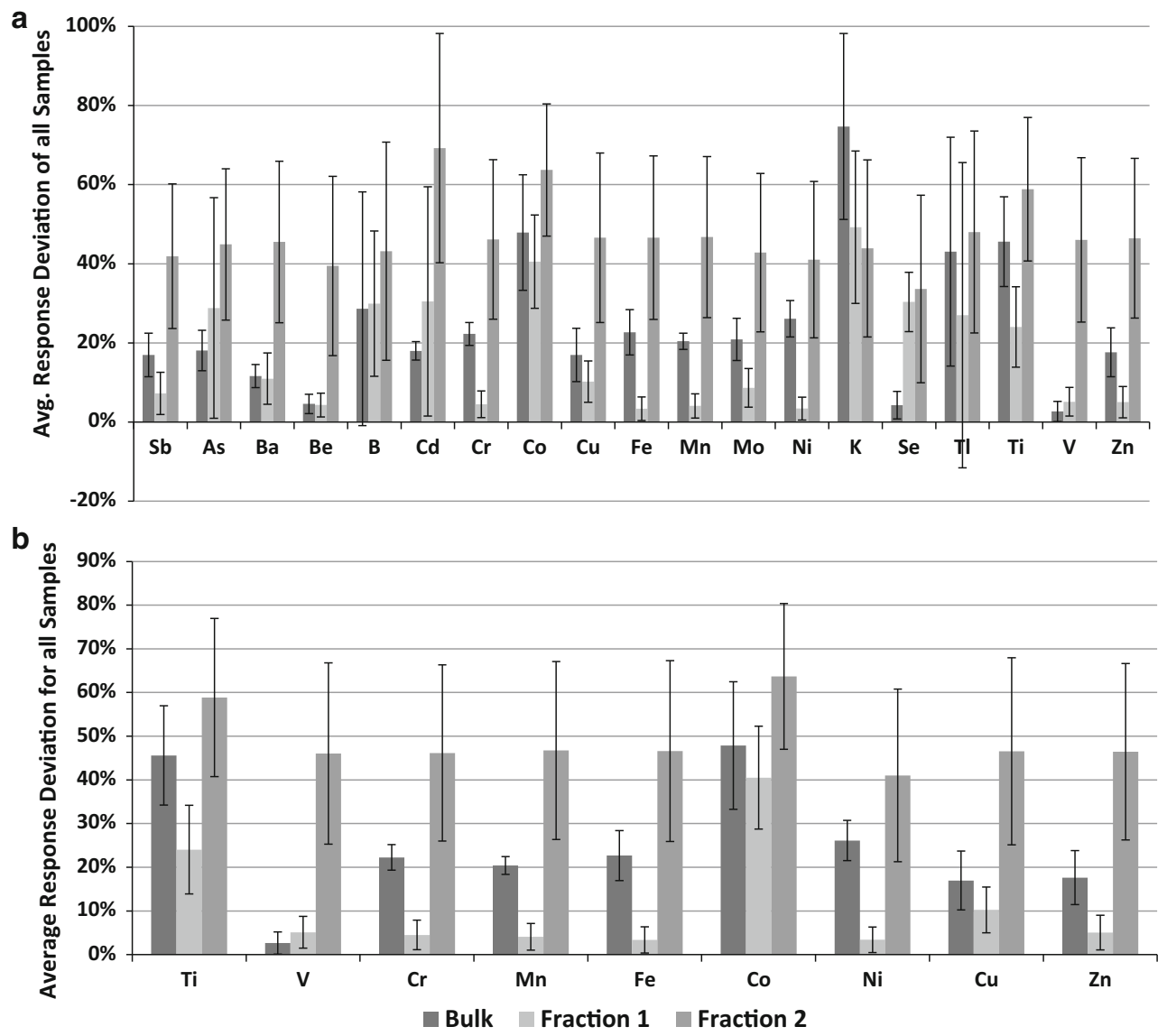

Fig. 4 Average of the absolute response deviation from $100 \%$ for a each metal and $\mathbf{b}$ first-row $d$-block metals

sporadic, with PW71 showing a $50 \%$ decrease in response and PW77, PW78, and PW97 each showing an increase of greater than $100 \%$. These responses also show that the samples with the highest turbidity were among the lowest matrix effects observed. This was also observed for potassium, thallium, and copper. Zinc and cadmium each show positive Spearman correlations, 0.465 and 0.642 , respectively, between analyte response and turbidity as the analytes approach $100 \%$ response. After SPE, the majority of samples showed decreased matrix effects for these five metals mentioned, but independent of the initial solution's turbidity. Suspended solids will be removed from the Fraction 1 solution after passing through the SPE column, indicating that another physical property of the solution has a greater positive effect in response accuracy than that lost by reduced turbidity.

\section{Metal summaries}

The emission responses for arsenic in the Bulk solutions were reasonably uniform throughout all twenty samples, ranging from 70.6 to $89.8 \%$. From a previous study involving a matrix of $0.1 \mathrm{M}$ of either $\mathrm{Na}, \mathrm{K}, \mathrm{Ca}$, or $\mathrm{Mg}$, measurements for arsenic revealed a recovery response ranging between 72 and $93 \%$, while the mixture of these four elements to the same $0.1 \mathrm{M}$ concentration gave a synergistic decrease in signal ranging from 50 to $67 \%$ (Pereira et al. 2012). Analysis of arsenic for Fraction 1 of the given samples resulted in a net increase in response, ranging from 104.8 to $179.7 \%$. When considering the conclusions by Pereira et al. (2012), it is suspected that a combination of $\mathrm{Na}, \mathrm{K}, \mathrm{Ca}$, and $\mathrm{Mg}$ may be removed by the SPE sorbent, causing less emission suppression for arsenic. At least one of these four elements resulted in reduced recoveries for each of the samples after being passed through the RP-SPE. It has been shown by others that metals can be deposited and accumulate onto $\mathrm{C} 18$ silica support, supporting this hypothesis (Engelhardt and Lobert 1999).

Beryllium shows no matrix effects present for the Bulk and Fraction 1 samples across all twenty well water samples. Beryllium is not expected to form oxides during residence time in the plasma, a chemical interference for which other metals are more susceptible and is well known to induce signal suppression. All but five samples show noticeable matrix effects in Fraction 2. 


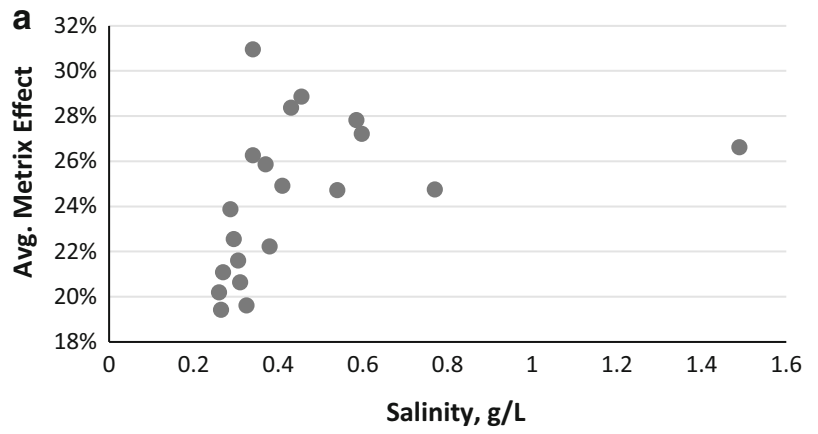

b

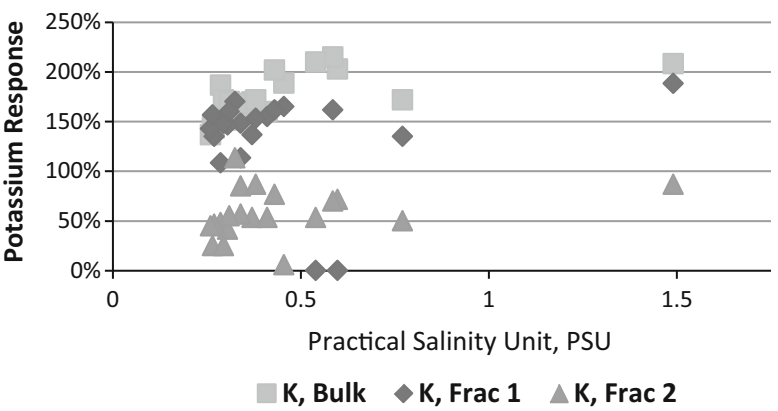

Fig. 5 Salinity influence for the observed matrix effects for a all samples as a function of averaged matrix effect of all metals and $\mathbf{b}$ the response of potassium under the three preparation approaches

Resulting responses for boron were quite varied across the twenty wells. Thirteen Bulk solution samples showed no matrix effects, but after SPE, only 3 of those 13 still showed no matrix effects. Fourteen samples showed an improvement in response for the Fraction 1 measurement, while three showed a decrease in response of the Bulk solution to Fraction 1 measurements. Of the Bulk measurements, the four reference wells had the greatest degree of matrix effect present. This effect was resolved in three of the reference samples, yielding a response between 80 and $100 \%$ in Fraction 1. All four of the reference samples were of the nearest samples to ideal response, while half of the remaining samples yielded an increased response near $150 \%$ of the standards.

Cadmium showed a matrix effect present in only three wells in the Bulk solution, but only to a slight degree with the greatest effect being PW9 with $78 \%$ response. Fraction 1 showed a reduction in sample matrix, except for in PW71, PW77, PW78, and PW97. The response change for each of these wells ranged from a Bulk response of at least $79 \%$ to a Fraction 1 response of less than $27 \%$. PW77 and PW78 are in near proximity to each other, being located in neighboring small counties on the southwest portion of our sampling region.

Potassium showed positive matrix effects in all Bulk samples, with the greatest degree having over $200 \%$ response. Fraction 1 reduced the positive matrix effects, but still, all except PW9 (108 \%) and PW74 (113\%) displayed these positive effects. It is not until Fraction 2 that the source for the response enhancement is removed from all samples. The SPE-retained matrix in Fraction 2 results in response suppression in all samples except PW41, PW50, PW55, and PW102. The significant difference between the matrices of Fraction 2 and that of Bulk or Fraction 1 shows that Fraction 2 contains no ionic species. These results indicate that in general, ions present in the samples gave rise to the enhanced sensitivity of the $\mathrm{K}$ measurements. Figure $5 \mathrm{~b}$ reveals a relationship between the solution salinity and responses in Bulk and Fraction 1, but a loss in salinity influence in Fraction 2. Nebulized droplets of a solution with a high ionic matrix will reach the Rayleigh limit quicker than less charged droplets, inducing an explosion of smaller droplets to evaporate more easily, resulting in more usable droplets reaching the plasma than with a matrix-free solution, e.g., standard solution (Agatemor and Beauchemin 2011). A spectral interference would give rise to the increased emission, but no interferences are listed near the $\mathrm{K}$ monitored wavelength of $766.49 \mathrm{~nm}$.

Selenium revealed a consistent response across all wells in the Bulk measurements, having all responses between 80 and $110 \%$. All wells increased response in Fraction 1, 15 of which improved to over $120 \%$ the calibration response, reported as a positive matrix effect. The reconstituted matrix of Fraction 2 reduced the response for all wells below $100 \%$ except for PW50, which was further enhanced to $155 \%$ response. Only 6 of the 20 wells in Fraction 2 showed no matrix effects.

Zinc showed moderate response inhibition across all twenty Bulk samples with an average response of $82.4 \%$ with eight wells exhibiting matrix effects. Treatment of the sample via SPE resulted in improved responses for all wells except PW97 which showed only a $1.6 \%$ response deviation in Bulk and an $11.1 \%$ response deviation in Fraction 1.

\section{Conclusion}

This study investigated the analyte-specific tendencies of the groundwater matrix from the Barnett Shale region of North Texas. Data show that the resulting matrix does not affect each elemental analyte to the same degree. For many metals, the matrix effect in Bulk sample solutions is considerable, while it is negligible for other metals. The use of reversed-phase solid-phase extraction to remove hydrophobic organic molecules reduced the sample matrix compared to Bulk for almost all measured samples, resulting in $74 \%$ of all measured metals in all samples showing no matrix effects. These results indicate that there is an effect from the hydrophobic constituents of the water 
when the Bulk was measured without pretreatment beyond simple acidification. This notion can be confirmed by Fraction 2, the retained matrix, which, when isolated, has quite unpredictable effects on each metal in each collected sample. Even though these samples were collected from two aquifers in North Texas, each sample was found to have varying unknown constituents through calculating a degree of matrix effect. Wells within the localized area of the same county still showed different characteristics of response impact. In general, all metals, except arsenic, sampled from the twenty samples across North Texas revealed either reduction or minimal change in matrix effect after RP-SPE treatment. Collectively, these data indicate that it is important to optimize sample preparation depending on the analyte of interest, for each and every sample to account for unknown or unanticipated matrix effects, unless a standard addition protocol can be used to ensure quantification is carried out in the exact same matrix as the sample of interest.

Acknowledgments Funds used to support this study were from the unrestricted and unrelated Young Investigator Award in Analytical Chemistry Award to the corresponding author from Eli Lilly and Company. We wish to thank the Shimadzu Center for Advanced Analytical Chemistry at the University of Texas at Arlington for the use of the ICP-OES instrumentation. With greatest importance, we continue to thank the participating well owners for their contributions to this research and others that have culminated through time.

\section{References}

Agatemor C, Beauchemin D (2011) Matrix effects in inductively coupled plasma mass spectrometry: a review. Anal Chim Acta 706:66-83

Barth S (1997) Comparison of NTIMS and ICP-OES methods for the determination of boron concentrations in natural fresh and saline waters. Fresenius J Anal Chem 358:854-855

Caenn R, Darley HCH, Gray GR (2011) Composition and properties of drilling and completion fluids. Gulf Professional Publishing, Waltham

Dettman JR, Olesik JW (2012) Reduction of matrix effects in quantitative and semi-quantitative inductively coupled plasmaoptical emission spectrometry using a partial local thermodynamic equilibrium model and an internal standard. Spectrochim Acta B 76:96-108

Dow Water and Process Solutions (2011) FILMTEC reverse osmosis membranes: technical manual. 609-00071-1009

Engelhardt H, Lobert T (1999) Chromatographic determination of metallic impurities in reversed-phase HPLC columns. Anal Chem 71:1885-1892

Ferreira SLC, de Andrade JB, Korn MGA, Pereira MG, Lemos VA, Santos WNL, Rodrigues FM, Souza AS, Fereira HS, Silva EGP (2007) Review of procedures involving separation and preconcentration for the determination of cadmium using spectrometric techniques. J Hazard Mater 145:358-367

Fink JK (2011) Petroleum engineer's guide to oil field chemicals and fluids. Gulf Professional Publishing, Waltham

Fontenot BE, Hunt LR, Hildenbrand ZL, Carlton DD Jr, Oka H, Walton JL, Hopkins D, Osorio A, Bjorndal B, Hu QH, Schug KA
(2013) An evaluation of water quality in private drinking water wells near natural gas extraction sites in the barnett shale formation. Environ Sci Technol 47:10032-10040

Fraser MM, Beauchemin D (2009) Evidence supporting the occurrence of Coulomb fission during conventional sample introduction in inductively coupled plasma mass spectrometry. J Anal At Spectrom 24:469-475

Groh S, Garcia C, Murtazin A, Horvatic V, Niemax K (2009) Local effects of atomizing analyte droplets on the plasma parameters of the inductively coupled plasma. Spectrochim Acta B 64:247-254

Jiang C (1996) Solubility and solubility constant of barium sulfate in aqueous sodium sulfate solutions between 0 and $80{ }^{\circ} \mathrm{C}$. J Solut Chem 25:105-111

Knápek J, Komárek J, Novotný K (2010) Determination of cadmium, chromium and copper in high salt samples by LA-ICP-OES after electrodeposition-preliminary study. Microchim Acta 171:145-150

Kramida A, Ralchenko Y, Reader J, NIST ASD Team (2013) NIST atomic spectra database (ver. 5.1). http://physics.nist.gov/asd

Nam S-H, Chung H, Kim J-J, Lee Y-I (2008) Mass background spectra of ICP-MS with various acids. Bull Korean Chem Soc 29:2237-2240

Nguyen HP, Li L, Nethrapalli IS, Guo N, Toren-Allerand CD, Harrison DE, Astle CM, Schug KA (2011) Evaluation of matrix effects in analysis of estrogen using liquid chromatographytandem mass spectrometry. J Sep Sci 34:1781-1787

Olesik JW (1991) Elemental analysis using ICP-OES and ICP/MS: An evaluation and assessment of remaining problems. Anal Chem 63:12A-21A

Olesik JW (1996) Fundamental research in ICP-OES and ICPMS. Anal Chem 68:469A-474A

Osborn SG, Vengosh A, Warner NR, Jackson RB (2011) Methane contamination of drinking water accompanying gas-well drilling and hydraulic fracturing. Proc Natl Acad Sci USA 108:8172-8176

Pereira CD, Aguirre MÁ, Nóbrega JA, Hidalgo M, Canals A (2012) Correction of matrix effects for As and Se in ICP OES using a flow blurring multiple nebulizer. $J$ Anal At Spectrom 27:2132-2137

PerkinElmer Inc. (2013) Atomic spectroscopy - a guide to selecting the appropriate technique and system. http://www.perkinelmer. com/CMSResources/Images/44-74482BRO_ WorldLeaderAAICPMSICPMS.pdf

Reedy RC, Scanlon BR, Walden S, Strassberg G (2011) Naturally occurring groundwater contamination in Texas. Final Report 1004831125; Texas Water Development Board

Sadler DA, Sun F, Howe SE, Littlejohn D (1997) Comparison of procedures for correction of matrix interferences in the multielement analysis of soils by ICP-AES with a CCD detection system. Mikrochim Acta 126:301-311

Sah RN, Brown PH (1997) Boron determination-a review of analytical methods. Microchem J 56:285-304

Silva JCJ, Baccan N, Nóbrega JA (2003) Analytical performance of an inductively coupled plasma optical emission spectrometer with dual view configuration. J Braz Chem Soc 14:310-315

Stewart II, Olesik JW (1998) Transient acid effects in inductively coupled plasma optical emission spectrometry and inductively coupled plasma mass spectrometry. J Anal At Spectrom 13:843-854

Stewart II, Olesik JW (1999) Droplet introduction to investigate space-charge effects in plasma mass spectrometry. J Am Soc Mass Spectrom 10:159-174

Stüber M, Reemtsma T (2004) Evaluation of three calibration methods to compensate matrix effects in environmental analysis with LC-ESI-MS. Anal Bioanal Chem 378:910-916

Texas Water Development Board (2014) Groundwater database reports. http://www.twdb.state.tx.us/groundwater/data/gwdbrpt.asp 
Thomsen V, Schatzlein D (2006) The laws of spectrochemistry. Spectroscopy 21:44-48

Thomsen V, Schatzlein D, Mercuro D (2006) Interelement corrections in spectrochemistry. Spectroscopy 23:32-40

US EPA (1999) Compendium of ERT groundwater sampling procedures, EPA/540/P-91/007
US EPA (2007) Inductively coupled plasma-atomic emission spectrometry. Method-6010C

Welch AH, Westjohn DB, Helsel DR, Wanty RB (2000) Arsenic in ground water of the United States: occurrence and geochemistry. Ground Water 38:589-604 